\title{
Synthesis and characterization of multifunctional hybrid-polymeric nanoparticles for drug delivery and multimodal imaging of cancer
}

This article was published in the following Dove Press journal:

International Journal of Nanomedicine

15 September 2015

Number of times this article has been viewed

\author{
Danny Jian Hang Tngl,* \\ Peiyi Songl,* \\ Guimiao Lin ${ }^{2,3, *}$ \\ Alana Mauluidy \\ Soehartono' \\ Guang Yang' \\ Chengbin Yang' \\ Feng Yin' \\ Cher Heng $\operatorname{Tan}^{4}$ \\ Ken-Tye Yong' \\ 'School of Electrical and Electronic \\ Engineering, Nanyang Technological \\ University, Singapore; ${ }^{2}$ The \\ Engineering Lab of Synthetic Biology, \\ ${ }^{3}$ Research Institute of Uropoiesis \\ and Reproduction, School of \\ Medicine, Shenzhen University, \\ Shenzhen, People's Republic of China; \\ ${ }^{4}$ Department of Diagnostic Radiology, \\ Tan Tock Seng Hospital, Singapore \\ *These authors contributed equally \\ to this work
}

Correspondence: Guimiao Lin

The Engineering Lab of Synthetic Biology, School of Medicine, Shenzhen University,

Shenzhen, People's Republic of China

Tel +867558667 1973

Email gmlin@szu.edu.cn

Ken-Tye Yong

School of Electrical and Electronic

Engineering, Nanyang Technological

University, 50 Nanyang Avenue,

Singapore 639798

Tel +65 67905444

Email ktyong@ntu.edu.sg
Abstract: In this study, multifunctional hybrid-polymeric nanoparticles were prepared for the treatment of cultured multicellular tumor spheroids (MCTS) of the PANC-1 and MIA $\mathrm{PaCa}-2$ pancreatic carcinoma cell lines. To synthesize the hybrid-polymeric nanoparticles, the poly lactic-co-glycolic acid core of the particles was loaded with Rhodamine $6 \mathrm{G}$ dye and the chemotherapeutic agent, Paclitaxel, was incorporated into the outer phospholipid layer. The surface of the nanoparticles was coated with gadolinium chelates for magnetic resonance imaging applications. This engineered nanoparticle formulation was found to be suitable for use in guided imaging therapy. Specifically, we investigated the size-dependent therapeutic response and the uptake of nanoparticles that were $65 \mathrm{~nm}, 85 \mathrm{~nm}$, and $110 \mathrm{~nm}$ in size in the MCTS of the two pancreatic cancer cell lines used. After 24 hours of treatment, the MCTS of both PANC-1 and MIA PaCa-2 cell lines showed an average increase in the uptake of $18.4 \%$ for both $65 \mathrm{~nm}$ and $85 \mathrm{~nm}$ nanoparticles and $24.8 \%$ for $110 \mathrm{~nm}$ nanoparticles. Furthermore, the studies on therapeutic effects showed that particle size had a slight influence on the overall effectiveness of the formulation. In the MCTS of the MIA PaCa- 2 cell line, $65 \mathrm{~nm}$ nanoparticles were found to produce the greatest therapeutic effect, whereas $12.8 \%$ of cells were apoptotic of which $11.4 \%$ of cells were apoptotic for $85 \mathrm{~nm}$ nanoparticles and $9.79 \%$ for $110 \mathrm{~nm}$ nanoparticles. Finally, the study conducted in vivo revealed the importance of nanoparticle size selection for the effective delivery of drug formulations to the tumors. In agreement with our in vitro results, excellent uptake and retention were found in the tumors of MIA PaCa-2 tumor-bearing mice treated with $110 \mathrm{~nm}$ nanoparticles.

Keywords: nanoparticles, individualized, cancer, multicellular tumor spheroids, hybridpolymeric

\section{Introduction}

Human cancers result in high mortality rates ${ }^{1}$ due to the challenges faced in early detection ${ }^{2,3}$ and treatment. ${ }^{4}$ Although the majority of cancers initially respond to drug therapy, disease recurrence and metastasis are the primary causes of mortality among cancer patients. ${ }^{5}$ Current treatments for managing cancer recurrence or metastases rely on the nonselective delivery of chemotherapeutic agents to the tumor sites. ${ }^{6}$ However, such approaches are ineffective in treating the disease due to their toxic effects in normal tissues. Therefore, the need to engineer customizable drug therapies for a more personalized treatment of cancer is urgent and would lead to more specific treatments, allowing the individualized delivery of anticancer drugs to tumors and maximizing the therapeutic effects of the drugs. Another challenge faced in the treatment of cancer is the evolving nature of cancer biology throughout the course of treatment. ${ }^{7}$ Therefore, a successful evaluation of potential therapies relies upon the concomitant development 
of new imaging modalities that enable the tumor response to the treatment to be monitored in real time. With the aid of the multimodal imaging tools, the drug formulation therapy can be adjusted accordingly as the disease progresses to different states. However, the currently available advanced imaging modalities, such as magnetic resonance imaging (MRI), positron emission tomography, computed tomography, and optical imaging, all have various limitations, such as sensitivity, resolution, and depth profiling. ${ }^{8,9}$ A single imaging modality is therefore unlikely to provide conclusive information on a particular therapeutic response. In this regard, the development of multimodal imaging tools is important to enable the cross-validation of the therapeutic effect of the drug formulations administered. ${ }^{10}$ Thus, the development of individualized drug delivery platforms that can be used in conjunction with multimodal imaging is crucial to successfully combat human cancers.

Polymer-based nanoparticles have been shown to have a high degree of customizability and are thus suitable candidates for use in the individualized treatment of cancer. ${ }^{11}$ Such particles exhibit many adjustable characteristics, such as their size, ${ }^{12}$ zeta potential, ${ }^{13}$ morphology, ${ }^{14}$ drug loading, ${ }^{15}$ and surface functionality. ${ }^{16}$ Many studies have shown that these characteristics play key roles in determining the outcome of cancer treatments. ${ }^{17}$ For example, targeted delivery can be achieved by functionalizing the nanoparticle surface with ligands that exhibit specific binding efficiency to the tumor cells. ${ }^{18,19}$ In addition, the nanoparticle surface can be modified using contrast agents, such as organic dyes ${ }^{20}$ or radioisotopes, for multimodal imaging. ${ }^{21}$ More importantly, both hydrophobic and hydrophilic drugs can also be incorporated in these nanoparticles for the effective therapy of cancers. For instance, Bisht et $\mathrm{al}^{22}$ showed that such polymeric nanoparticles provide an excellent drug delivery vehicle for hydrophobic reagents, such as curumin, and the formulation showed significant anticancer effects. Similarly, Kim et $\mathrm{al}^{23}$ presented a polymeric nanomedicinal platform loaded with ferric oxide nanocrystals and doxorubicin for use in simultaneous cancer-targeted imaging and drug delivery. $\mathrm{Yu}$ et $\mathrm{al}^{24}$ reported that a preparation of polymeric hybrid nanoparticles comprising an outer polymer coating and an inner superparamagnetic iron oxide core could be used for tumor targeting and therapy in vivo.

Recent studies have shown that customizable nanoparticles have a great potential for the delivery of drug therapy to cancers by exploiting their size-dependent uptake and their ability to target the tumor tissues. ${ }^{12,25,26}$ In this study, we show that newly engineered multifunctional hybrid-polymer nanoparticles can be used for the delivery of therapeutic reagents and the imaging of cancer both in vitro and in vivo. The synthesized nanoparticles had cores made of poly lactic-co-glycolic acid (PLGA), ${ }^{27,28}$ which are widely used for drug delivery applications. ${ }^{29,30}$ It should be noted that PLGA tends to degrade hydrolytically into nontoxic byproducts within 50-60 days in the body. ${ }^{31,32}$ The PLGA core was encapsulated with a phospholipid outer shell made of methoxy-poly(ethylene glycol)-1,2-distearoyl-sn-glycero3-phosphoethanolamine-N (mPEG-DSPE) molecules. ${ }^{33-35}$ Moreover, both PLGA and mPEG-DSPE have been approved as chemical materials for pharmaceutical use by the US Food and Drug Administration, ${ }^{35,36}$ giving such nanoparticles great potential for future clinical developments. The PLGA core designed here was loaded with hydrophilic Rhodamine $6 \mathrm{G}$ to allow the uptake and penetration of the different sizes of nanoparticles to be monitored using fluorescent imaging. ${ }^{37,38}$ The PLGA core was then encapsulated with a phospholipid micelle to promote the solubility of the nanoparticle in the biological buffers. This also increased the bioavailability of the particles, enabling them to evade capture by the reticuloendothelial system. ${ }^{39}$ The outer shell of the micelle region was loaded with Paclitaxel, a potent hydrophobic anticancer drug, for cancer therapy purposes. ${ }^{40}$ To achieve multimodal imaging, the micelle surface was further functionalized with gadolinium chelates for MRI. By changing the molar ratio of PLGA and mPEG-DSPE, different sizes of nanoparticles could be prepared, allowing the synthesis of the optimal nanoparticle size for specific in vitro or in vivo applications. Unlike traditional cell monolayers, the MCTS models displayed more tumor-like features, allowing a better prediction of the in vivo tumor responses to different drug treatments. ${ }^{41}$ Nanoparticle uptake and penetration studies using the MCTS model revealed that each cell line had a specific affinity toward different sizes of nanoparticles. For instance, we found that the MIA PaCa-2 cell line displayed the strongest affinity for $110 \mathrm{~nm}$ nanoparticles compared with $65 \mathrm{~nm}$ and $85 \mathrm{~nm}$ nanoparticles. Further therapeutic studies using the nanoparticles suggested that particle size had a slight effect on the overall therapeutic effect of the formulation. In the MCTS of the MIA PaCa-2 cell line, $65 \mathrm{~nm}$ nanoparticles produced the greatest therapeutic effect, of which $12.8 \%$ of cells were apoptotic; $11.4 \%$ of cells were apoptotic using $85 \mathrm{~nm}$ nanoparticles and $9.79 \%$ using $110 \mathrm{~nm}$ nanoparticles after 24 hours of treatment. These studies highlight that $110 \mathrm{~nm}$ nanoparticles are suitable for use as multifunctional probes in in vivo imaging and therapy applications. As a proof of concept, in vivo studies were performed on MIA 
PaCa-2 tumor-bearing mice treated with $65 \mathrm{~nm}, 85 \mathrm{~nm}$, and $110 \mathrm{~nm}$ hybrid-polymeric nanoparticles. When the mice were administered with $110 \mathrm{~nm}$ nanoparticles, the tumor exhibited high nanoparticle retention, which was consistent with the in vitro studies of the MCTS model. In addition, the drug molecules were gradually released into the tumor site, allowing an effective drug delivery therapy in vivo. MRI imaging of the ex vivo tumor tissues revealed that the nanoparticles had indeed accumulated in the tumor cells as shown by the T1-weighted imaging process. The strength of multimodal imaging was therefore clearly shown from these in vivo experiments, in which fluorescence and MRI techniques were used to verify the distribution of nanoparticles in the tumor. Our results highlight the potential of using customizable multifunctional nanoparticle formulations for the individualized treatment of cancer in the future. Such approaches could potentially revolutionize the treatment of cancer, allowing the maximization of therapeutic effects from the nanoformulation and concomitantly minimizing the side effects caused during drug therapies.

\section{Materials and methods Cell culture}

Human prostate cancer cell line PC-3 and human pancreatic cancer cell lines PANC-1 and MIA PaCa-2 were purchased from the American Type Culture Collection (ATCC, Manassas, VA, USA). Penicillin-Streptomycin $100 \mu \mathrm{g} / \mathrm{mL}$, and Ham's F-12K (Kaighn's) Medium were purchased from Gibco (Life Technologies, SG, Singapore). Dulbecco's Modified Eagle's Medium ([DMEM], Hyclone) and fetal bovine serum ([FBS], Hyclone) were purchased from Thermo Fisher Scientific. PANC-1 cells were maintained in a culture with DMEM, supplemented with $10 \%$ FBS, Penicillin-Streptomycin $(100 \mu \mathrm{g} / \mathrm{mL})$. MIA PaCa-2 cells were cultured in DMEM supplemented with $10 \%$ FBS, 2.5\% horse serum (ATCC), $100 \mu \mathrm{g} / \mathrm{mL}$ penicillin, and streptomycin. PC-3 cells were cultured in F-12K with $10 \%$ FBS, Penicillin-Streptomycin $(100 \mu \mathrm{g} / \mathrm{mL}) .2 \times 10^{3}$ cells were resuspended in medium ( $5 \mathrm{~mL}$ per $70 \mathrm{~mm}$ dish). The cultures were maintained at $37^{\circ} \mathrm{C}$ in a humidified atmosphere with $5 \% \mathrm{CO}_{2}$ inside an incubator.

\section{Culturing of multicellular spheroid tumors}

The preparation of the multicellular tumor spheroids (MCTS) was performed using the hanging droplet method. ${ }^{42,43}$ For each cell line, a single cell suspension was obtained from the cell cultures prepared in 2.1 after each culture had reached $90 \%$ confluence. Using a hemacytometer, the cell concentration within the cell suspension then adjusted by adding the appropriate medium until a concentration of $2 \times 10^{4}$ cells $/ \mathrm{mL}$ was reached. Next, with a pipette, 25 drops of $20 \mu \mathrm{L}$ of the cell suspension were deposited onto the lid of a $70 \mathrm{~mm}$ Petri dish in a $5 \times 5$ matrix. Approximately $5 \mathrm{~mL}$ of phosphate buffered saline (PBS) was put into the base of the Petri dish, and the lid was then inverted and used to cap the Petri dish base. The hanging drop cultures were then maintained in a humidified incubator at $37^{\circ} \mathrm{C}$ with $5 \% \mathrm{CO}_{2}$. After an incubation period of 4 days, cellular aggregation occurred and MCTS were formed. The mean tumor size used was $263,119 \pm 26,032 \mu \mathrm{m}^{2}$.

\section{Preparation of multifunctional hybrid- polymer nanoparticles}

50:50 Poly(DL-lactide/glycolide) copolymer (PLGA), molecular weight (MW) 150,000 Da was purchased from Polysciences (Taipei, Taiwan), mPEG-DSPE MW 2,000 Da, mPEG-DSPE amine (mPEG-DSPE-NH ${ }_{2}$ ), methoxy-poly(ethylene-glycol)-thiol MW 2,000 Da and methoxypoly(ethylene glycol)-succinimidyl succinate MW 2,000 Da were purchased from Laysan Bio, Inc. (Arab, AL, USA). Rhodamine 6G, gadolinium(III) chloride hexahydrate and chloroform were purchased from Sigma Aldrich (Singapore). Paclitaxel was obtained from TCI Chemicals (Tokyo, Japan). 1,4,7,10-Tetraazacyclododecane-1,4,7,10-tetraacetic acid mono ( $N$-hydroxysuccinimide ester) (DOTA-NHS-ester) was purchased from Macrocyclics (Dallas, TX, USA). PLGA was dissolved in chloroform solvent to make up a concentration of $80 \mathrm{mg} / \mathrm{mL}$ together with an appropriate concentration of Paclitaxel and Rhodamine 6G, forming an organic solution. An aqueous phospholipid solution was then prepared with a 5:1 molar ratio of $\mathrm{mPEG}-\mathrm{DSPE}$ and $\mathrm{mPEG}-\mathrm{DSPE}-\mathrm{NH}_{2}$ to $1 \mathrm{mg} / \mathrm{mL}$ concentration. The organic solution was then added dropwise to the aqueous solution under sonication with a tip sonicator (Sonic Dismembrator, ultrasonic Processor FB-505, Thermo Fisher Scientific). The addition of the organic solution stopped when the target ratio of PLGA/DSPE was reached. Six different PLGA/DSPE ratios were used such as $0.05,0.1,0.2,0.4,0.8$, and 1.6. The PLGA/DSPE ratios were varied by adjusting the amount of PLGA, while keeping mPEG-DSPE constant at $1 \mathrm{mg} / \mathrm{mL}$. The resulting nanoparticle mixture was then put into a vacuum desiccator at $28^{\circ} \mathrm{C}$ for 12 hours to evaporate the excess chloroform. After which, surface modification of the mPEG-DSPE-NH amine groups was achieved by adding DOTA-NHS-ester in excess $(1 \mathrm{mg} / \mathrm{mL})$ at $\mathrm{pH} 8.5$ and incubating for 48 hours 
at $28^{\circ} \mathrm{C}$. Next, gadolinium was added in excess $(1 \mathrm{mg} / \mathrm{mL})$ at $\mathrm{pH} 8.0$ and incubated for 48 hours at $28^{\circ} \mathrm{C}$. Finally, the nanoparticle mixture was purified for 72 hours, undergoing four cycles of dialysis against PBS pH 7.4 with molecular weight cutoff (MWCO) 12,000 Da dialysis tubing.

\section{Particle size and zeta potential characterization}

Mean particle size was measured using dynamic light scattering (DLS). DLS and zeta potential were determined using the NanoBrook 90Plus Particle Size Analyzer (Brookhaven Instruments, Holtsville, NY, USA). Each measurement was performed in triplicate, and a total of four different samples were measured for each data point. Both particle sizing and zeta potential measurements were performed at $10 \times$ dilution with deionized (DI) water.

\section{Rhodamine 6G and Paclitaxel loading measurement}

Ultraviolet visible (UV-Vis) spectroscopy was performed using quartz cuvettes to measure the absorption of the nanoparticle solutions with reference to DI water (UV-Vis Spectrometer UV-2450, Shimadzu Instruments). The degree of absorption was measured at $530 \mathrm{~nm}$ and $227 \mathrm{~nm}$ for Rhodamine 6G and Paclitaxel, respectively. The concentration of each component was then calculated using the BeerLambert Law.

\section{Imaging nanoparticles with scanning electron microscopy and transmission electron microscopy}

For scanning electron microscopy (SEM), the nanoparticle solutions to be examined were diluted four times and then $10 \mu \mathrm{L}$ droplets were placed onto a silicon wafer. The droplets were then left to dry completely within a vacuum desiccator. After which, it was sputter coated with a thin layer of gold, and then the particles were imaged under an SEM (LEO 1550 Gemini). For transmission electron microscopy (TEM), the nanoparticle solutions were diluted two times and then $10 \mu \mathrm{L}$ droplets were placed onto TEM copper grids and left to dry in a vacuum desiccator. The particles were then imaged under TEM (JEOL JEM 2010/F) at $100 \mathrm{kV}$ accelerating potential and imaged with a GATAN Multiscan CCD camera.

\section{Nuclear magnetic resonance TI relaxation characterization}

Nanoparticle solutions of four different sizes $(100 \mathrm{~nm}$, $90 \mathrm{~nm}, 80 \mathrm{~nm}, 75 \pm 2 \mathrm{~nm}$ ) were prepared in four different dilutions of mPEG-DSPE ( $1 \mathrm{mg} / \mathrm{mL}, 0.5 \mathrm{mg} / \mathrm{mL}, 0.25 \mathrm{mg}$ / $\mathrm{mL}$, and $0.125 \mathrm{mg} / \mathrm{mL}$ ), with DI water for characterization of their T1 relaxation time by using phantom imaging (Siemens Magnetron Trio, Siemens Healthcare, Erlangen, Germany). A spin-echo inversion recovery sequence was used with a field of view of $250 \times 250 \mathrm{~mm}$, matrix size of $128 \mathrm{p} \times 128 \mathrm{~s}$, recovery time of 3,000 $\mathrm{ms}$ and inversion times of 100-3,200 ms. Images were acquired using a flex coil and at a magnetic field strength of $3 \mathrm{~T}$.

\section{In vitro fluorescence uptake studies of nanoparticles into spheroids}

Three different sizes ( $65 \mathrm{~nm}, 85 \mathrm{~nm}, 110 \mathrm{~nm})$ of nanoparticle formulations were prepared as mentioned in Section "Preparation of multifunctional hybrid-polymer nanoparticles" with equal concentrations of Rhodamine $6 \mathrm{G}(0.5 \mu \mathrm{g} / \mathrm{mL})$. Five microliters of each of the prepared nanoparticle solutions were then administered to each of the $20 \mu \mathrm{L}$ droplets, which contain one formed spheroid each. The spheroids were then maintained in a humidified incubator at $37^{\circ} \mathrm{C}$ with $5 \% \mathrm{CO}_{2}$. The bright field and fluorescence images of spheroid cultures were obtained by using an inverted microscope (Nikon Eclipse Ti-U) at $10 \times$ magnification. This procedure was repeated for the three examined cell lines, PANC-1, MIA PaCa-2, and PC-3. The spheroids were imaged at 1 hour, 2 hours, 3 hours, 6 hours, and 24 hours after administration of the nanoparticle formulation. Fluorescence images were obtained using a filter set with excitation $525 \mathrm{~nm}$ and emission $555 \mathrm{~nm}$ (Chroma Technology Corp, Bellows Falls, VT, USA). Image analysis was performed with the microscope vendor software. Single factor analysis of variance at the 95\% significance level was used to analyze the statistical significance of the results.

\section{In vitro spheroid treatment studies}

Nanoparticle formulations were prepared as mentioned in the section "Preparation of multifunctional hybrid-polymer nanoparticles" with approximately equal concentrations of Rhodamine 6G $(0.5 \mu \mathrm{g} / \mathrm{mL})$ and Paclitaxel $(137.5 \pm 22.7 \mathrm{nM})$ to make nanoparticles of three different sizes $(65 \mathrm{~nm}, 85 \mathrm{~nm}$, and $110 \mathrm{~nm})$. For the control studies, similar nanoparticle formulations $(65 \mathrm{~nm}, 85 \mathrm{~nm}$, and $110 \mathrm{~nm})$ were prepared and tested. Five microliters of each of the prepared nanoparticle solutions were then administered to each of the $20 \mu \mathrm{L}$ droplets, which contain one formed spheroid each. The spheroids were then maintained in a humidified incubator at $37^{\circ} \mathrm{C}$ with $5 \% \mathrm{CO}_{2}$. After 24 hours of incubation, the spheroids were harvested by washing with PBS off the lids of the Petri dishes. The spheroids were then centrifuged at 4,000 rpm 
for 4 minutes to concentrate the cells. Propidium iodide (PI) in DMSO $(2 \mu \mathrm{L} / \mathrm{mL})$ was then added to the spheroids, and a pipette was used to homogenize the cell suspension solution and create a single cell suspension. The mixture was then left to incubate for 30 minutes without exposure to light and then PI and DMSO were removed through centrifugation, and the cells were resuspended in PBS. Flow cytometry was then performed with an excitation and emission filter set of $540 \mathrm{~nm}$ and $620 \mathrm{~nm}$, respectively, for PI as the marker. Optical compensation was performed with single-stained compensation control samples, where the cells were stained with only Rhodamine $6 \mathrm{G}$ and another with only PI.

\section{In vitro release studies}

In vitro release profiles of Paclitaxel from the fabricated nanoparticles were investigated in an aqueous medium containing PBS. Three sized $65 \mathrm{~nm}, 85 \mathrm{~nm}$, and $110 \mathrm{~nm}$ nanoparticles, with equal concentrations of $1 \mathrm{mg} / \mathrm{mL}$ DSPE and 137.5 $\pm 22.7 \mathrm{nM}$ Paclitaxel were investigated. One milliliter ( $1 \mathrm{mg} / \mathrm{mL}$ concentration) of each nanoparticle solution was sealed into a dialysis membrane bag (MWCO 12,000 Da). Each dialysis bag was then placed into a $20 \mathrm{~mL}$ glass bottle containing $19 \mathrm{~mL}$ of PBS solution under slight agitation. At preset time intervals, $1 \mathrm{~mL}$ of the PBS solution was withdrawn for UV-Vis spectroscopy in quartz cuvettes to measure the absorption of the nanoparticle solutions with reference to PBS solution (UV-Vis Spectrometer UV-2450, Shimadzu Instruments). The absorption spectra were measured and monitored for Paclitaxel at $227 \mathrm{~nm}$ and for Rhodamine 6G at $530 \mathrm{~nm}$. The concentrations of Paclitaxel and Rhodamine $6 \mathrm{G}$ can be estimated using the Beer-Lambert Law. After each measurement, the PBS within the samples was replaced with $19 \mathrm{~mL}$ of fresh PBS solution. This procedure was repeated at $4^{\circ} \mathrm{C}$ to determine the release of Paclitaxel and Rhodamine $6 \mathrm{G}$ during storage. For the release studies using FBS, $1 \mathrm{mg}$ of nanoparticles were incubated in $20 \mathrm{~mL}$ of $10 \% \mathrm{FBS}$ solution. At predetermined time intervals, $1 \mathrm{~mL}$ of solution was withdrawn, and the nanoparticles were then pelleted using ultracentrifugation at 15,000 rpm. The pellet was then purified by removing the supernatant and washed twice with DI water and resuspended into a solution for analysis to determine the amount of Paclitaxel and Rhodamine 6G released.

\section{In vivo studies of nanoparticle retention}

Athymic nude mice (BALB/cASlac-nu, female) of 5-6 weeks of age were obtained from Shanghai Slac Laboratory Animal, Chinese Academy of Sciences (Licensing number: SCXK2007-0005). Human cell line experiments were approved by the Nanyang Technological University Biological Safety Committee (BPN-0001-2012-EEE) and the Office of Health and Safety (RA_EEE_5528). Animal research was carried out in compliance with the National Standard of Animal Care and Use Procedures (20080820). The mice were kept in an isolated biosafety facility for specific pathogen free (SPF) animals with bedding, food, and water. Tumor growth was monitored every 24-48 hours until these tumors exceeded $100-150 \mathrm{~mm}^{3}$ in volume ( $\sim 10-15$ days posttransplantation of cells). After which, the mice were administered with subcutaneous injections near the site of the tumors with $100 \mu \mathrm{L}$ of nanoparticle solution $(1 \mathrm{mg} / \mathrm{mL})$. Three mice were used, and each mouse was injected with a nanoparticle solution containing particles of different sizes such as $65 \mathrm{~nm}, 85 \mathrm{~nm}$, and $110 \mathrm{~nm}$. After injection, mice were anesthetized with $50 \mu \mathrm{L}$ pelltobarbitalum natricum $(10 \mathrm{mg} / \mathrm{mL})$ per injection. Then the mice were imaged using the IVIS Lumina II in vivo optical imaging system (Perkin Elmer, Waltham, MA, USA; excitation: $480 \mathrm{~nm}$, emission: $555 \mathrm{~nm}$ ) at specific time points ( 1 hour, 3 hours, and 48 hours after injection), to monitor biodistribution of the nanoparticles. After 48 hours of observation, the mice were anesthetized, and the tumors were removed for MRI under a magnetic field of $3 \mathrm{~T}$ using a flex coil (Siemens Magnetron Trio, Siemens Healthcare, Erlangen, Germany). A total of four sequences were used: T1-weighted spin-echo, T1-weighted spin-echo fat-saturation, T2-weighted turbo spin-echo, and T2-weighted turbo spinecho fat-saturation. For T1-weighted measurements, an echo time of $10 \mathrm{~ms}$, recovery time of $414 \mathrm{~ms}$, field of view of $200 \times 200 \mathrm{~mm}$, and matrix size of $192 \mathrm{p} \times 250$ s were used. For T2-weighted measurements, an echo time of $87 \mathrm{~ms}$, recovery time of $3,381 \mathrm{~ms}$, field of view of $200 \times 200 \mathrm{~mm}$, and matrix size of $336 \mathrm{p} \times 448 \mathrm{~s}$ were used.

\section{Results and discussion Synthesis and characterization of hybrid- polymeric nanoparticles}

The hybrid-polymeric nanoparticles comprised of an inner core of PLGA, which encapsulated Rhodamine 6G dye. This core was surrounded by an outer micelle shell into which the drug Paclitaxel was incorporated. The nanoparticles were prepared by a single-step sonication method modified from Fang et al. ${ }^{44}$ The micelle shell was made up of a mixture of mPEG-DSPE and mPEG-DSPE-NH ${ }_{2}$ phospholipids. Using the $\mathrm{NH}_{2}$ functional group, surface functionalization of the particle was carried out using DOTA-NHS ester. DOTA then functioned as a chelating agent to bind gadolinium MRI contrast agent to the surface of the nanoparticle. 
A schematic representation of this is given in Figure 1E. The size of the nanoparticles could be tailored by manipulating the ratio of PLGA and DSPE that was added to the reaction mixture. Figure 1 shows the hydrodynamic diameter of the nanoparticles prepared (Figure 1A). We found that the nanoparticle size increased as the molar ratio of PLGA/ DSPE increased. The smallest nanoparticle with the size of $70 \pm 7.44 \mathrm{~nm}$ was obtained with a low PLGA/DSPE ratio of 0.05 , and largest size of $114 \pm 5.11 \mathrm{~nm}$ was obtained using a PLGA/DSPE ratio of 1.6. The morphology of the fabricated nanoparticles was examined using SEM (Figure 1F-K). The particles prepared using lower PLGA/DSPE ratios (Figure 1F) appeared to be rod shaped and have an average width and length of $35 \mathrm{~nm}$ and $110 \mathrm{~nm}$, respectively. When the PLGA/ DSPE ratio was increased, larger and more spherical particles were formed, as shown in Figure 1G-I. The morphology of the nanoparticles underwent another distinct change when the size approached $200 \mathrm{~nm}$. As observed in Figure 1J and $\mathrm{K}$, an irregular shape was observed and most of the particles appeared to have a rough boundary surface. TEM was also used to examine the internal structure of the fabricated nanoparticles. As seen in Figure 1M, a relatively dense region was observed in the center region of the particles. Conversely, when no PLGA was added, no dense region was observed (Figure $1 \mathrm{~N}$ ), indicating that the PLGA core was encapsulated within the mPEG-DSPE micelle. The loading efficiency of Rhodamine $6 \mathrm{G}$ within the nanoparticles was determined by using UV-Vis spectroscopy. The increment of Rhodamine $6 \mathrm{G}$ concentration within the nanoparticles was nonlinear, with higher concentrations of Rhodamine 6G being entrapped in the particle as the PLGA/DSPE ratio increased. The same characterization method was performed for Paclitaxel encapsulated in the nanoparticles. The concentration of Paclitaxel remained relatively constant at $137.5 \pm 22.7 \mathrm{nM}$, regardless of the PLGA/DSPE ratio used in the synthesis process, which was to be expected because the mPEG-DSPE concentration was kept constant at $1 \mathrm{mg} / \mathrm{mL}$. As reported by Ip et al, ${ }^{45}$ such a synthesis process would first create multilayered lipid layers embedded with the hydrophobic drugs. PLGA, when added to this system, would be encapsulated by the micelles, forming a single-layered micelle system. These processes would explain why the Paclitaxel concentration remained constant within a nanoparticle system at a constant concentration of mPEG-DSPE. The zeta potential is an important factor in maintaining the colloidal stability of particle dispersion. ${ }^{46}$ In our study, the larger nanoparticles had zeta potential values ranging from $-40.0 \mathrm{mV}$ to $-46.9 \mathrm{mV}$ and smaller nanoparticles had values ranging from $-22.5 \mathrm{mV}$ to
$-30.7 \mathrm{mV}$. The surface of the nanoparticles was then further functionalized with gadolinium chelates, thereby allowing the particles to exhibit contrast enhancement for MRI. A T1 relaxometry study revealed no significant differences in $\mathrm{T} 1$ relaxation time between nanoparticles of different sizes. It should be noted that gadolinium chelates are directly associated with the DSPE-NH ${ }_{2}$ molecule, and thus, the major factor that would determine relaxation time would be the total amount of DSPE-NH $\mathrm{N}_{2}$ available within a nanoparticle. As shown in Figure 1D, nanoparticles prepared with a higher concentration of DSPE-NH ${ }_{2}(0.167 \mathrm{mg} / \mathrm{mL})$ exhibited a mean relaxation time of $967.3 \mathrm{~ms}$. In contrast, a lower concentration $(0.04 \mathrm{mg} / \mathrm{mL})$ of DSPE- $\mathrm{NH}_{2}$ resulted in a mean relaxation time of $1,442.5 \mathrm{~ms}$, suggesting that an effective concentration of at least $0.04 \mathrm{mg} / \mathrm{mL}$ DSPE- $\mathrm{NH}_{2}$ is required to obtain good contrast enhancement for MRI. Finally, it has been suggested that fluorescence dyes loaded in a bare PLGA core may migrate to the cellular membranes upon contact. ${ }^{47}$ In our study, the PLGA core was encapsulated in an outer phospholipid shell, which prevented loaded core reagents from diffusing outward. ${ }^{48}$ In addition, the outer shell contained outward facing PEG chains (Figure 1E), preventing direct contact between the lipid layers of the nanoparticles and the outer membrane of a cell and blocking the adsorption of the dye into the cells. ${ }^{49}$ Other studies performed with similar nanoparticle structures have shown a strong association of the loaded dyes with the nanoparticles. ${ }^{50}$

\section{In vitro study of uptake and penetration of nanoparticles}

The cellular uptake and penetration of nanoparticle treatments are important parameters to be considered when designing the physicochemical property of the nanoparticle because they will determine the overall therapeutic action on the tumor cells. In this study, multifunctional nanoparticles of different sizes, $65 \mathrm{~nm}(\sigma=4.85), 85 \mathrm{~nm}(\sigma=6.57)$, and $110 \mathrm{~nm}(\sigma=9.94)$, were tested in the MCTS (Figure 2) of different cell lines. Additional information on the size distribution of the prepared nanoparticle formulations is provided in the supporting information (Figure S1). Fluorescence imaging analysis was then performed for each MCTS models at different time intervals. To date, it has been shown that the penetration and uptake of nanoparticles in tumor tissue can be due to several factors. In our study, the uptake and penetration of nanoparticles into the tumor spheroids were probably a result of the interstitial flow of nanoparticles into the tumor matrix. ${ }^{25}$ Basically, a relatively high density of nanoparticles was surrounded by the tumor interfaces. Thereafter, the receptor-mediated endocytosis 


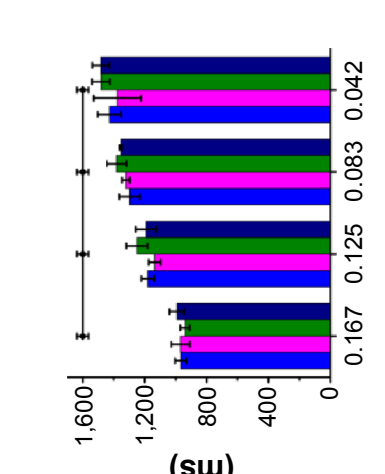

(su)

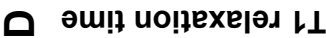
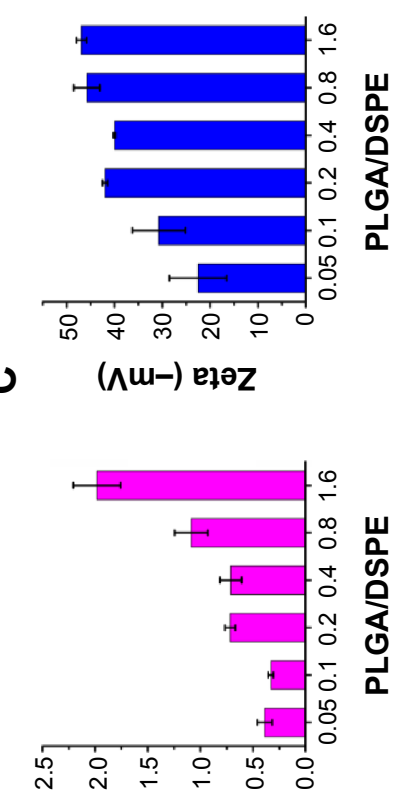

(ךu/6n)

m 99 әи!шерочу

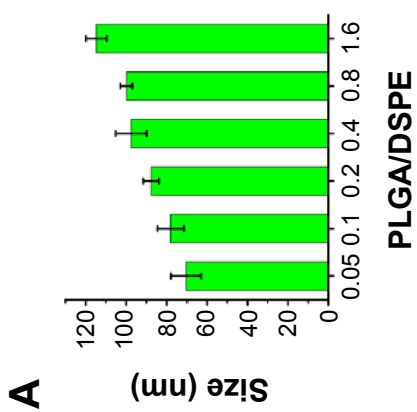

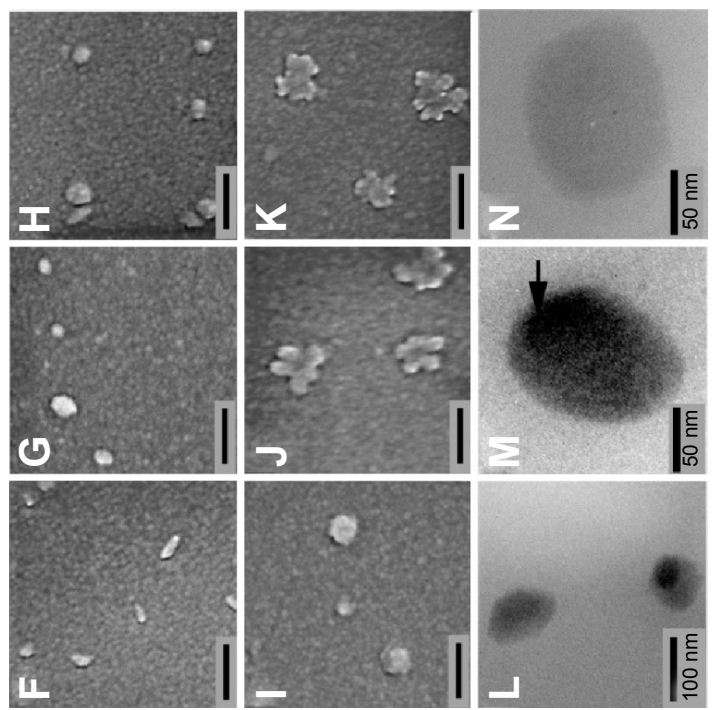

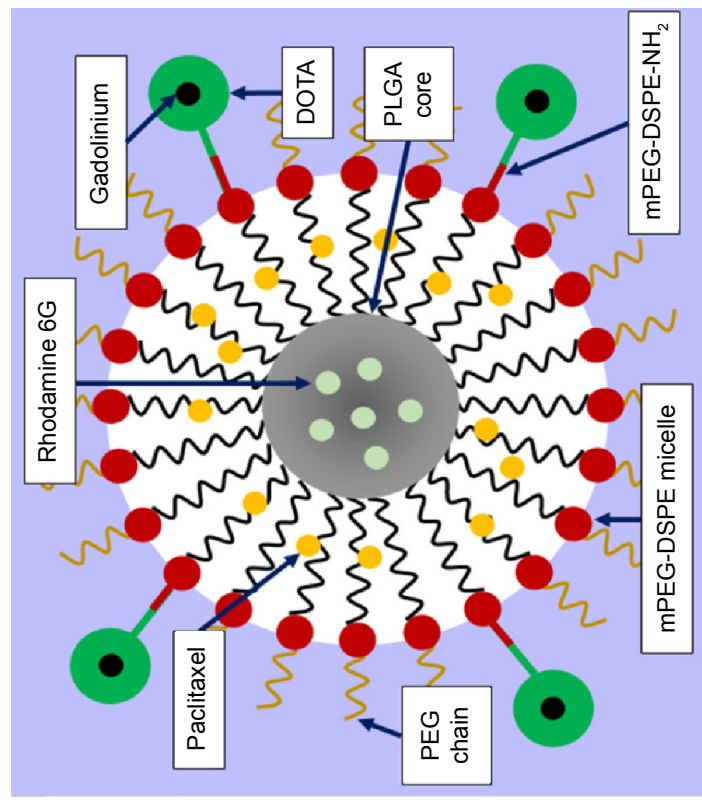

ш

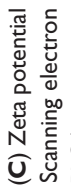

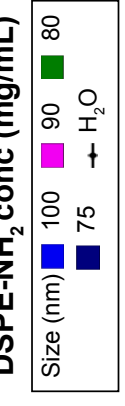

它产产

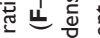

幽它

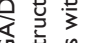

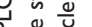

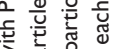

긍 웜ำ

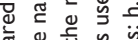

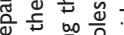

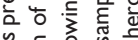

选

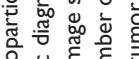

证施它

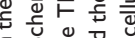

$\subseteq$ 员讨

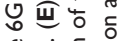

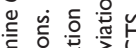

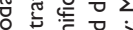

눙

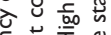

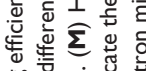

总施

ฮั

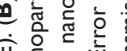

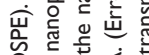

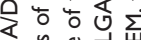

บे

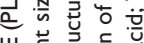

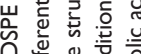

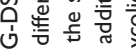

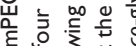

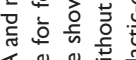

తิ

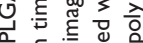

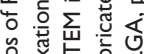

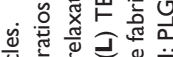

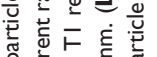

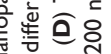

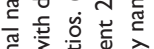

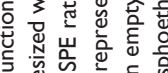

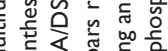

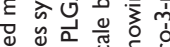

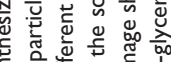

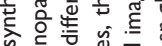

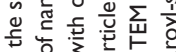

\&

ᄃ 论

可它

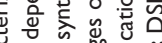

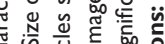

$\checkmark$

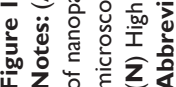


A

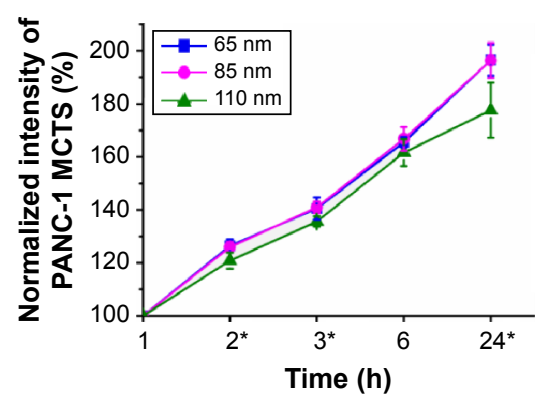

$65 \mathrm{~nm}$

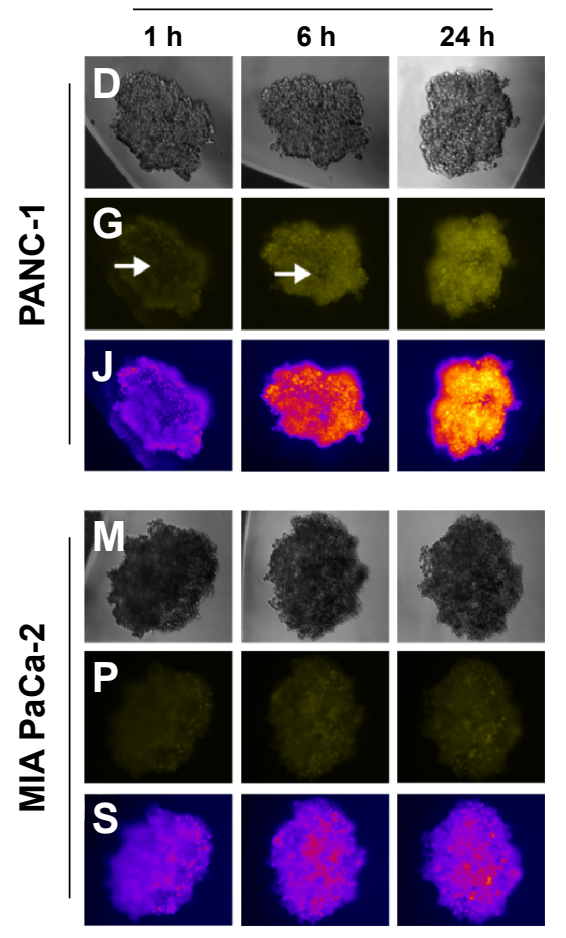

B

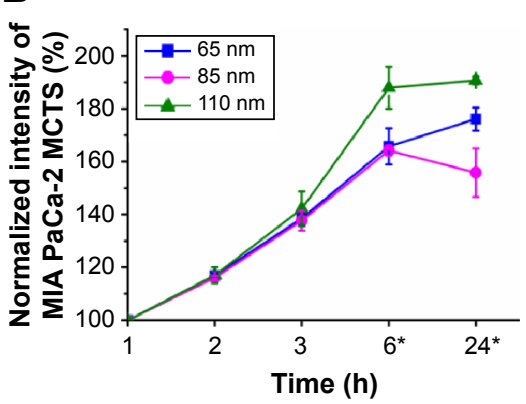

C

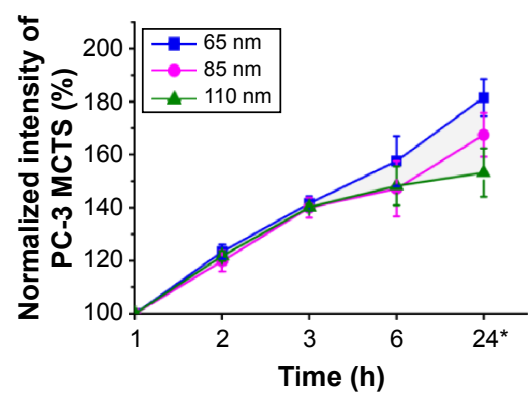

$110 \mathrm{~nm}$
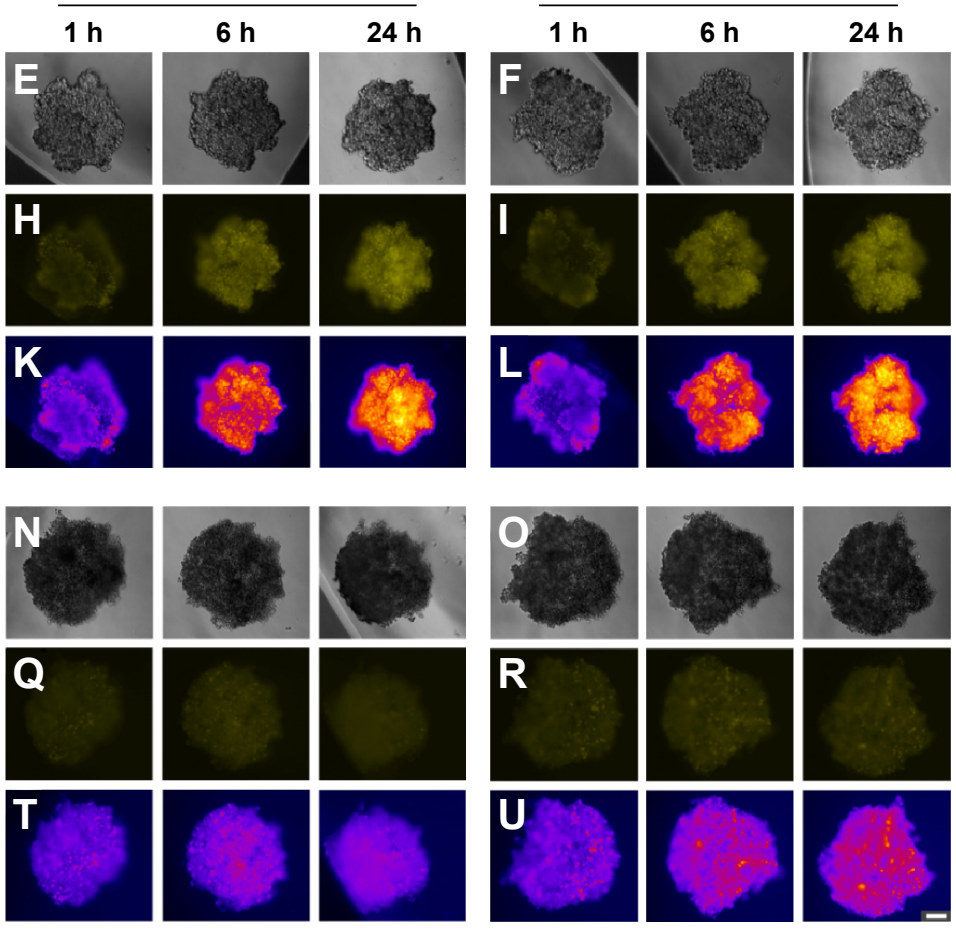

Relative fluorescent intensity (au)

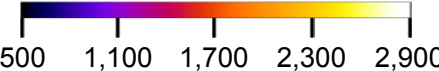

Figure 2 Uptake of different sizes of nanoparticles $(65 \mathrm{~nm}, 85 \mathrm{~nm}$, and $110 \mathrm{~nm})$ in the MCTS of the PANC-I, MIA PaCa-2, or PC-3 cell lines.

Notes: (A) Normalized relative fluorescence intensity of the MCTS of the PANC-I cell line at different time intervals. (B) Normalized relative fluorescence intensity of the MCTS of the MIA PaCa-2 cell line at different time intervals. (C) Normalized relative fluorescence intensity of the MCTS of the PC-3 cell line at different time intervals. For the MCTS of the PANC-I cell line treated with $65 \mathrm{~nm}, 85 \mathrm{~nm}$, and II $10 \mathrm{~nm}$ nanoparticles at I hour, 6 hours, and 24 hours: (D-F) bright field images, (G-I) fluorescence images with the white arrow highlighting the uneven distribution of the nanoparticles, and $(\mathbf{J}-\mathbf{L})$ intensity maps showing the relative fluorescent intensity of different regions of the MCTS of PANC-I. For the MCTS of the MIA PaCa-2 cell line treated with $65 \mathrm{~nm}, 85 \mathrm{~nm}$, and II0 nm nanoparticles at I hour, 6 hours, and 24 hours: (M-O) bright field images, (P-R) fluorescence images, and (S-U) intensity maps showing the relative fluorescent intensity of different regions of the MCTS of MIA PaCa-2. The scale bar represents $100 \mu \mathrm{m}$. Error bars indicate the standard deviation and the number of samples used in each measurement $(n=4)$. *Indicates statistical difference $(P<0.05)$ amongst the three mean fluorescent measurements at that time point, obtained from the testing of the three nanoparticle solutions based on the single variable analysis of variance test.

Abbreviations: MCTS, multicellular tumor spheroids; PLGA, poly lactic-co-glycolic acid; DSPE, distearoyl-sn-glycero-3-phosphoethanolamine-N; h, hours.

pathway may have helped the nanoparticles to migrate into the tumor spheroid tissues. ${ }^{51}$ These pathways may be mediated by proteins, such as clathrin and dynamin, ${ }^{52}$ which may be absorbed onto the nanoparticle surface, thereby allowing them to be taken up by the tumor spheroids through the endocytosis process. Our data suggested that indeed each MCTS cell line displayed size-selective uptake and penetration for different sizes of nanoparticles.
In the MCTS of the PANC-1 cell line, $85 \mathrm{~nm}$ nanoparticles had the most favorable uptake and penetration. In the in vitro penetration studies (Figure 2G-I), the MCTS of the PANC-1 cell line revealed a non-uniform nanoparticle distribution within the first 6 hours of treatment for all sizes of nanoparticle administered, which was particularly obvious in the PANC-1 sample treated with $65 \mathrm{~nm}$ nanoparticles, as indicated in Figure 2G. The inner core 
region had a lower fluorescent intensity compared with the peripheral regions, indicating less nanoparticle accumulation. Even after 24 hours of treatment, the MCTS of the PANC-1 cell line treated with $65 \mathrm{~nm}$ (Figure $2 \mathrm{G}$ ) and $110 \mathrm{~nm}$ (Figure 2I) nanoparticles still showed a sizeable central region with low fluorescent intensity, indicating a low uptake of nanoparticles. For MCTS of the PANC-1 cell line treated with $85 \mathrm{~nm}$ nanoparticles (Figure $2 \mathrm{H}$ ), a similar trend was also observed after the first 6 hours of treatment. However, after 24 hours, the central regions had a much higher fluorescent intensity compared to the peripheral regions, indicating that the nanoparticles had successfully penetrated the core of the MCTS. The MCTS of the PANC-1 cell line was incubated with free Rhodamine $6 \mathrm{G}$ to serve as a positive control, and the results are provided in the supplementary material (Figure S2D). The data from the uptake studies of the different sizes of nanoparticles in the MCTS of the PANC-1 cell line further suggest that $85 \mathrm{~nm}$ is the optimum size for the treatment of the PANC-1 cell line (Figure 2A). The $85 \mathrm{~nm}$ nanoparticles showed the highest uptake, on a par with the $65 \mathrm{~nm}$ nanoparticles and $18.8 \%$ higher than that of $110 \mathrm{~nm}$ nanoparticles. The $85 \mathrm{~nm}$ nanoparticles thus had both optimum uptake and penetration characteristics for MCTS models of the PANC-1 cell line.

In the MIA PaCa-2 cell line, the nanoparticle uptake and penetration studies on the MCTS model show that $110 \mathrm{~nm}$ nanoparticles had the optimum uptake and penetration. In the nanoparticle uptake studies, an increase in average uptake of $24.8 \%$ was observed for $110 \mathrm{~nm}$ nanoparticles compared to that of the $65 \mathrm{~nm}$ and $85 \mathrm{~nm}$ nanoparticles (Figure 2B) after 24 hours of treatment. The penetration studies showed that MIA PaCa-2 MCTS exhibited a relatively uniform uptake throughout for all three particle sizes tested, as shown by the uniform fluorescent intensity of the images (Figure 2P-R). These results suggest that excellent uptake and penetration of nanoparticles in the tumor spheroids can be achieved using a particle size of $110 \mathrm{~nm}$. This size-selective uptake behavior characteristic has also been observed in other cell lines, such as PC-3, which is of prostate cancer origin. A similar uptake preference was observed for $65 \mathrm{~nm}$ nanoparticles in PC-3 MCTS (Figure 2C). These results suggest that nanoparticles size plays a key role for targeted imaging as well as therapeutic applications.

\section{Therapeutic effects of multifunctional nanoparticles in vitro}

The therapeutic effects of the prepared multifunctional nanoparticles on the MCTS of PANC-1 and MIA PaCa-2 cell lines were evaluated. The nanoparticles were loaded with the same concentration of Paclitaxel (137.5 $\pm 22.7 \mathrm{nM})$. From the in vitro release studies (Figure 3B), drug release from the nanoparticles showed an average gradual release of $30.4 \%$ of the total loaded drug over 24 hours for all three nanoparticle sizes dispersed in PBS. In addition, the in vitro release study was carried out in the presence of $10 \%$ FBS to examine its effects on the release of Paclitaxel and Rhodamine 6G from the nanoparticles. A more rapid release of Paclitaxel was observed, and more than $73 \%$ of the total loaded drug was found to be released over 24 hours for all three nanoparticle sizes. Rhodamine $6 \mathrm{G}$ was well protected within the PLGA inner core, and no release of the dye was observed over a 24-hour examination period. The release of Paclitaxel and Rhodamine $6 \mathrm{G}$ from the designed nanoparticles at $4^{\circ} \mathrm{C}$ was also examined and the results can be found in the supporting information (Figure S3).

The MCTS of the MIA PaCa-2 cell line were treated with the nanoparticle formulations for 24 hours, and the percentage of cells in the apoptotic state was determined. The percentage of apoptotic cells was directly proportional to the therapeutic effect of the prepared nanoparticle formulations. Flow cytometry analysis revealed that the Paclitaxel-loaded nanoparticles had effective therapeutic effects on the MCTS of the MIA PaCa-2 cell line. As seen in Figure 3A, in the control group of MIA PaCa-2 cells treated with unloaded Paclitaxel nanoparticles, a lower percentage of apoptotic cells $(1.66 \%)$ were observed. No significant differences in the percentage of cellular apoptosis was found in any of the control groups treated with $65 \mathrm{~nm}, 85 \mathrm{~nm}$, and $110 \mathrm{~nm}$ unloaded nanoparticles, suggesting that the unloaded nanoparticles had relatively low cytotoxicity compared to loaded particles. Thus, the average $11.3 \%$ increase in the percentage of apoptotic cells resulted from the treatment with Paclitaxelloaded nanoparticles. With regard to treatments with different nanoparticle sizes, the treated groups had $12.8 \%$ of apoptotic cells with $65 \mathrm{~nm}, 11.4 \%$ with $85 \mathrm{~nm}$, and 9.79\% with $110 \mathrm{~nm}$ nanoparticles compared with controls treated with nanoparticles without loaded Paclitaxel (Figure 3A). On average, a $2.18 \%$ increase in the percentage of apoptotic cells was observed for the MCTS of the MIA PaCa- 2 cell line treated with the $65 \mathrm{~nm}$ nanoparticles compared to those treated with $85 \mathrm{~nm}$ and $110 \mathrm{~nm}$ nanoparticles. Analyzing the detailed flow cytometry results (Figure $3 \mathrm{C}-\mathrm{F}$ ), the higher percentage of apoptosis caused by the $65 \mathrm{~nm}$ nanoparticle formulation was due to the increased count of the cells from the $7 \times 10^{3}$ to $8 \times 10^{3}$ fluorescent intensity region compared to the $85 \mathrm{~nm}$ and $110 \mathrm{~nm}$ nanoparticles. These experiments showed the potential of such hybrid-polymeric nanoparticles 
A

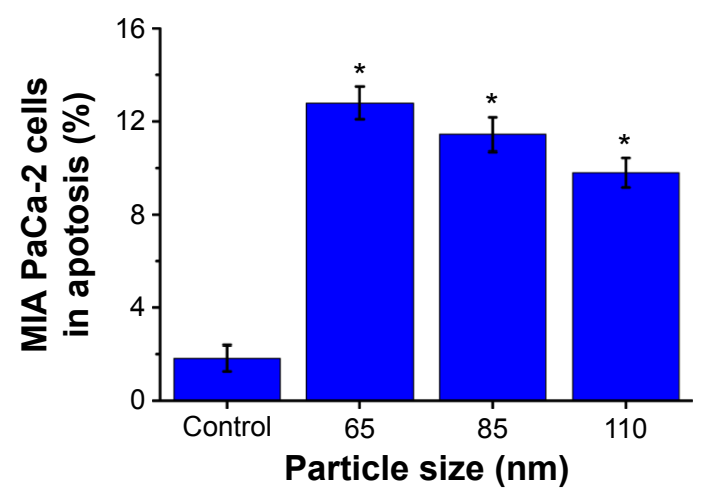

B

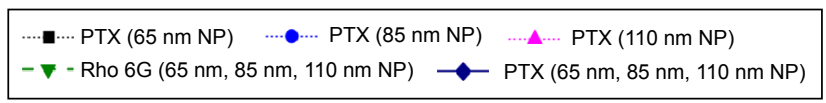

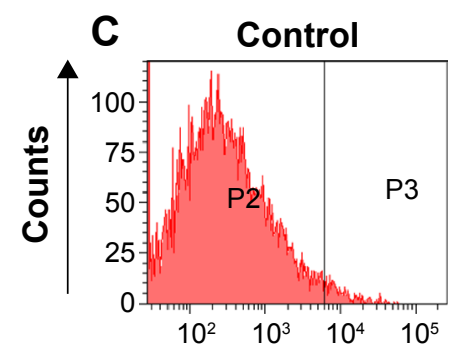
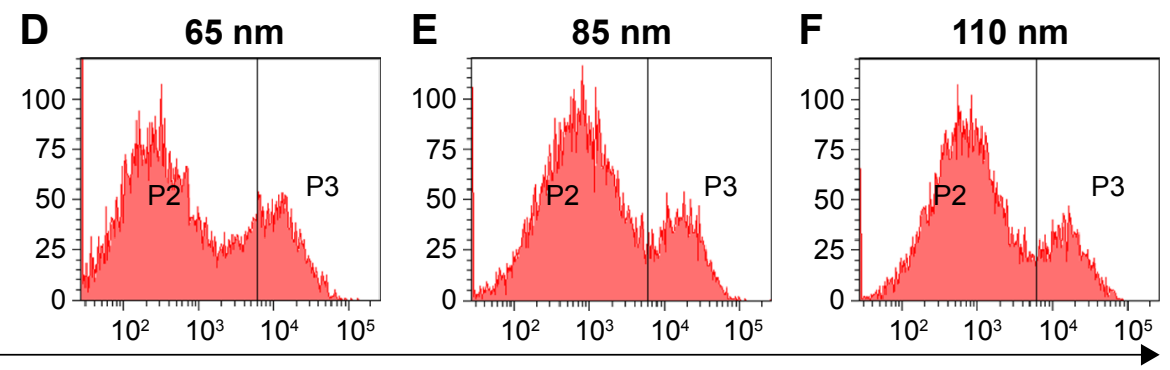

Propidium iodide fluorescent intensity (au)

Figure 3 Flow cytometry was used to investigate the relationship between the size and therapeutic effect of the multifunctional nanoparticles in the MCTS of the MIA $\mathrm{PaCa}-2$ cell line.

Notes: (A) The percentage of MIA PaCa-2 cells in apoptosis after 24 hours of treatment with nanoparticles of $65 \mathrm{~nm}, 85 \mathrm{~nm}$, and II $10 \mathrm{~nm}$. (B) In vitro release studies of the anticancer drug Paclitaxel (PTX) and Rhodamine 6G (Rho 6G) from $65 \mathrm{~nm}, 85 \mathrm{~nm}$, and II $10 \mathrm{~nm}$ nanoparticle formulations. Dotted lines and solid lines indicate in vitro studies performed in PBS and FBS, respectively. Rhodamine 6G release was examined in both PBS and FBS, represented by the dashed lines. (C-F) Flow cytometry results showing the cell count ( $y$-axis) and propidium iodide fluorescent intensity ( $x$-axis) of cells from MIA PaCa-2. (Vertical lines indicate the standard deviation and the number of samples used in each measurement, $n=4)$. *Indicates statistical difference $(P<0.05)$ using single variable analysis of variance $(A N O V A)$ between the three treated groups. \#Indicates statistical difference $(P<0.05)$ using single variable ANOVA between the Paclitaxel release from the different sizes of nanoparticles.

Abbreviations: MCTS, multicellular tumor spheroids; PBS, phosphate buffered saline; FBS, fetal bovine saline; PTX, Paclitaxel; h, hours; NP, nanoparticles.

for use in therapy and that the differences between the therapeutic effects of different sizes of nanoparticles were only slight.

\section{Multifunctional nanoparticles for multimodal imaging of tumors in vivo}

Our in vitro results showed that MIA PaCa-2 MCTS had a high preferential uptake of $110 \mathrm{~nm}$ nanoparticles. As a proof of concept, MIA PaCa-2 tumor-bearing nude mice were used to cross-check the trend observed in the in vitro models presented earlier. The $65 \mathrm{~nm}, 85 \mathrm{~nm}$, or $110 \mathrm{~nm}$ nanoparticles loaded with Rhodamine 6G, Paclitaxel, and gadolinium chelates were injected subcutaneously into the tumor in vivo. Subsequently, fluorescence imaging and MRI were performed to evaluate the retention of the nanoparticles within the tumors. Fluorescence imaging (Figure 4A-C) revealed that $65 \mathrm{~nm}$ and $85 \mathrm{~nm}$ nanoparticles were not detected in the tumor 1 hour after the injection, indicating the poor retention of these formulations by the tumors. In contrast, the $110 \mathrm{~nm}$ nanoparticles were retained in the tumor 1 hour after the injection and the fluorescence signals could still be detected after 48 hours of treatment. This florescence imaging result was consistent with the observations from the MIA PaCa-2 MCTS model. As reported by Cabral et al, ${ }^{53}$ polymeric nanoparticle accumulation within tumors in vivo are highly dependent on nanoparticle size and on the tumors formed by different cancer cell lines due to their individual morphology and vasculature characteristics.

For the MRI experiments, the tumors from the mice were harvested for T1- and T2-weighted sequences analysis. The T2-weighted images in Figure 4F show the rough morphology of the tumor samples represented by the dark areas at the center of the imaging tube. In contrast, the MRI of control solutions in Figure 4F and $\mathrm{G}$ remained dark, which is an important observation because the nanoparticle solutions had some fat content due to phospholipid molecules. 
A
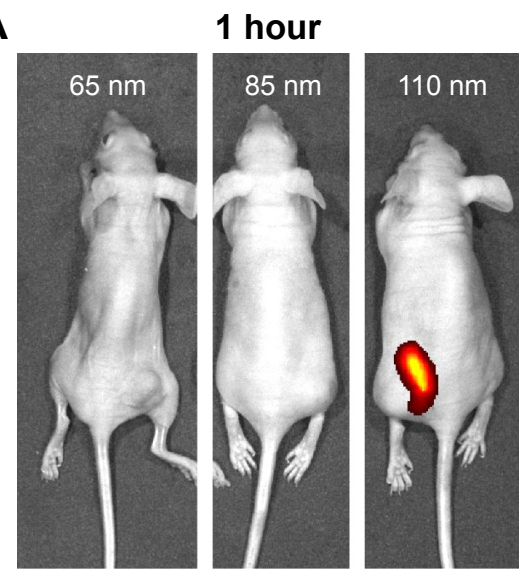

B

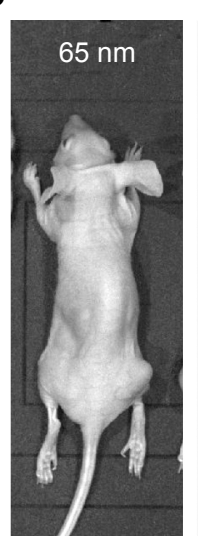

3 hours

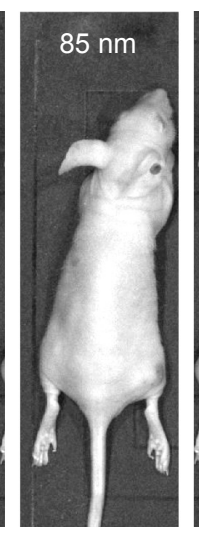

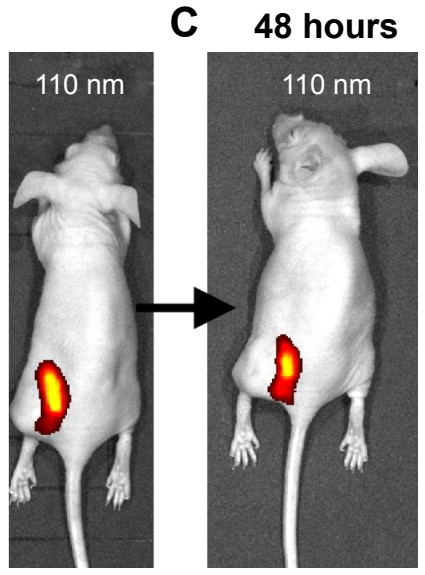

Radiant efficiency $\left(\times 10^{9}\right)$

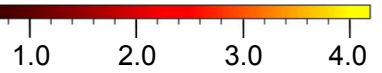

T1-weighted
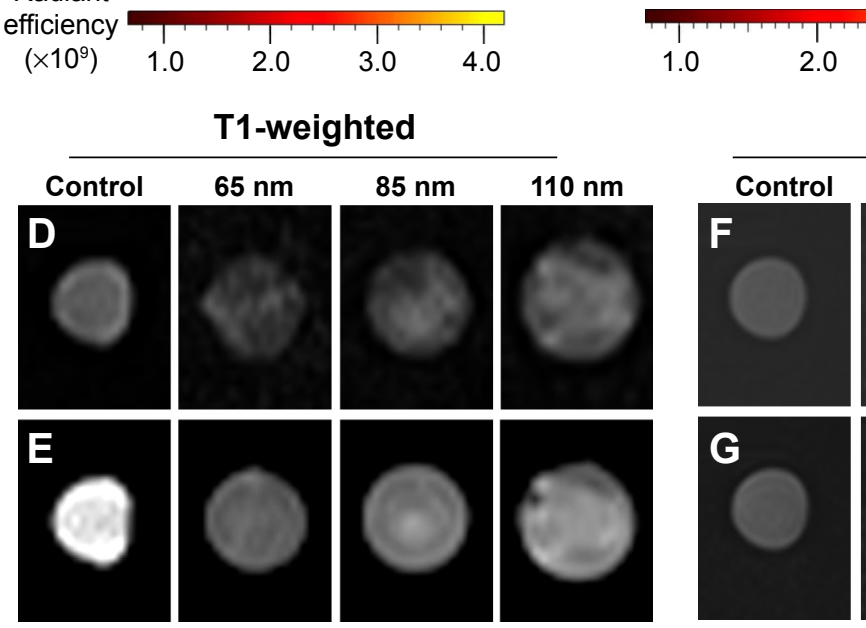

\section{T2-weighted}
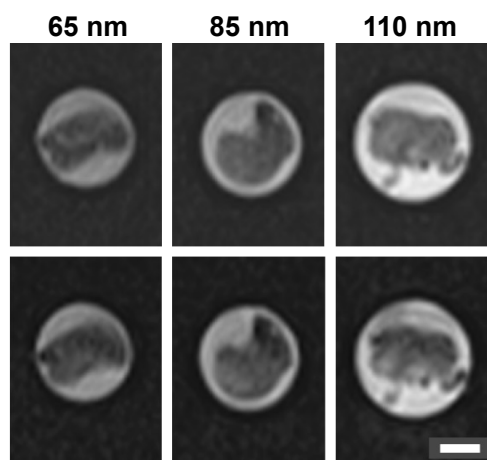

Figure 4 In vivo fluorescence and magnetic resonance imaging using the prepared multifunctional nanoparticles.

Notes: Fluorescence images taken for tumor-bearing mice treated with (A) $65 \mathrm{~nm}, 85 \mathrm{~nm}$, and II0 nm nanoparticles after I hour, (B) $65 \mathrm{~nm}, 85 \mathrm{~nm}$, and II0 nm nanoparticles after 3 hours, and (C) with $110 \mathrm{~nm}$ nanoparticles after 48 hours. (D) TI-weighted spin-echo images of extracted tumors. (E) TI-weighted fat saturation spin-echo images of extracted tumors. (F) T2-weighted turbo spin-echo images of extracted tumors. (G) T2-weighted fat saturation turbo spin-echo images of extracted tumors. Control samples comprised of I $\mathrm{mg} / \mathrm{mL}$ nanoparticle solutions. Radiant efficiency was measured using arbitrary units. Scale bar represents $\mathrm{I} 50 \mu \mathrm{m}$ for (B) and $400 \mathrm{~mm}$ for (D-G).

Since fatty tumors appear to be hypointense during T2 fat-saturated imaging, ${ }^{54}$ and the signal was too weak to be detected by the T2 imaging, any changes in the T2 fat-saturated images must have been caused by the presence of fat in the tissues. Fat-saturated T2-weighted images (Figure 4G) show no significant changes from the T2-weighted images (Figure 4F), indicating that the tumors consisted largely of dense and low-fat tissues. From the T1-weighted images in Figure 4D, the control solution sample comprising $1 \mathrm{mg} / \mathrm{mL}$ nanoparticles can be seen to exhibit a high contrast signal. Because the tumors lacked fatty tissues, as seen from the T2 fat-saturated images, the observed contrast from the tumors in the T1-weighted imaging resulted from the signal enhancement of the nanoparticles, indicating that the MRI contrast agent, gadolinium chelates, were successfully functionalized to the nanoparticles surface. In our MRI experiments, a minimal uptake of $65 \mathrm{~nm}$ and $85 \mathrm{~nm}$ nanoparticles in the tumors was observed. However, high contrast signals were obtained from tumors tissue harvested from mice treated with $110 \mathrm{~nm}$ nanoparticles, clearly suggesting that the $110 \mathrm{~nm}$ nanoparticles possessed a higher retention rate than that of the $65 \mathrm{~nm}$ and $85 \mathrm{~nm}$ nanoparticles and gave rise to the higher MRI contrast signals.

Our in vivo study showed consistency with the in vitro studies, demonstrating that the simultaneous imaging and therapeutic properties of multifunctional nanoparticles can be used to develop treatments for chronic diseases, such as cancer. Moreover, our studies also highlighted that hybridpolymeric nanoparticles are an excellent platform for drug delivery and the multimodal imaging of tumors. These nanoparticles can be uniquely tailored for a wide range of applications from targeted drug therapy to monitoring the 
progression of the tumor in vivo. Using their loading properties, they can be loaded with other smaller nanoparticles, such as magnetic ferric oxide nanocrystals, to leverage on their functionalities to perform MRI simultaneously with magnetically induced heat generation and drug delivery. ${ }^{55}$ Furthermore, the surface of the multifunctional nanoparticles presented here can be extensively modified for the potential treatment of other chronic illnesses, such as Alzheimer's disease, where the micelle surfaces functionalized with Pluronic block copolymers have been shown to enhance drug uptake in primary cultured bovine brain microvessel endothelial cell monolayers. ${ }^{56} \mathrm{We}$ envision that these hybrid polymeric nanoparticles will be useful for clinical applications in the near future. As previously discussed, our designed nanoparticles were derived from US Food and Drug Administration approved materials and were able to degrade into biologically inert byproducts. Through the incorporation of different contrast agents (eg, fluorescence and magnetic resonance) into the nanoparticles designed here, multimodal imaging can be carried out in real time to evaluate the biodistribution of nanoparticles in the body and thereby understand the effectiveness of the drug's efficacy in vivo.

\section{Conclusion}

In summary, hybrid-polymeric nanoparticles were fabricated for multimodal imaging and therapy of pancreatic cancer in vitro and in vivo. The synthesized nanoparticles comprised of a core made of PLGA, into which Rhodamine 6G fluorescent dye is loaded. This PLGA core is encapsulated by a phospholipid micelle, into which Paclitaxel, which is a potent anticancer drug for common cancer therapy use, was introduced. The micelle surface was functionalized with gadolinium chelates as an MRI contrast agent. Nanoparticle uptake and penetration studies showed that the two pancreatic cancer cell lines each had a preferential uptake of different sizes of nanoparticles. Specifically, the MIA PaCa-2 cell line showed the greatest preference for the uptake of $110 \mathrm{~nm}$ nanoparticles. Our therapeutic study showed that the developed nanoparticles could be used effectively to deliver therapeutic drugs and successfully cause the apoptosis of tumor cells. The tumors exhibited a high degree of retention with the $110 \mathrm{~nm}$ nanoparticle formulation, which was consistent with the in vitro study. Magnetic resonance imaging of the ex vivo tumor tissue confirmed the presence of nanoparticles in the tumor cells.

\section{Acknowledgments}

The presented study was supported by the the Nanyang Technological University-National Healthcare Group
Collaboration Grant (M4061202.040), partially by the Singapore Ministry of Education under Tier 2 Research Grant MOE2010-T2-2-010 (M4020020.040 ARC2/11) and partially by the Shenzhen Basic Research Project Grant (JCYJ20140418182819164). We would also like to extend special thanks to Ms Evelyn Quah Shi Shi and Ms Shirleen Eng for providing their expertise in the Magnetic Resonance Imaging of the tumor samples.

\section{Disclosure}

The authors report no conflicts of interest in this work.

\section{References}

1. Ward JF, Pagliaro LC, Pisters LL. Salvage therapy for radiorecurrent prostate cancer. Curr Probl Cancer. 2008;32(6):242-271.

2. Jacobs IJ, Menon U. Progress and challenges in screening for early detection of ovarian cancer. Mol Cell Proteomics. 2004;3(4): 355-366.

3. Etzioni R, Urban N, Ramsey S, et al. The case for early detection. Nat Rev Cancer. 2003;3(4):243-252.

4. Shannon AM, Bouchier-Hayes DJ, Condron CM, Toomey D. Tumour hypoxia, chemotherapeutic resistance and hypoxia-related therapies. Cancer Treat Rev. 2003;29(4):297-307.

5. Freedland SJ, Humphreys EB, Mangold LA, et al. Risk of prostate cancer-specific mortality following biochemical recurrence after radical prostatectomy. JAMA. 2005;294(4):433-439.

6. Brannon-Peppas L, Blanchette JO. Nanoparticle and targeted systems for cancer therapy. Adv Drug Deliv Rev. 2012;64(suppl 0):206-212.

7. Gottesman MM, Fojo T, Bates SE. Multidrug resistance in cancer: role of ATP-dependent transporters. Nat Rev Cancer. 2002;2(1):48-58.

8. Hoffman JM, Gambhir SS. Molecular imaging: the vision and opportunity for radiology in the future 1. Radiology. 2007;244(1):39-47.

9. Prasad PN. Introduction to Biophotonics. New York: John Wiley \& Sons; 2004.

10. Kim J, Piao Y, Hyeon T. Multifunctional nanostructured materials for multimodal imaging, and simultaneous imaging and therapy. Chem Soc Rev. 2009;38(2):372-390.

11. Ferrari M. Cancer nanotechnology: opportunities and challenges. Nat Rev Cancer. 2005;5(3):161-171.

12. Huang K, Ma H, Liu J, et al. Size-dependent localization and penetration of ultrasmall gold nanoparticles in cancer cells, multicellular spheroids, and tumors in vivo. ACS Nano. 2012;6(5):4483-4493.

13. Patil S, Sandberg A, Heckert E, Self W, Seal S. Protein adsorption and cellular uptake of cerium oxide nanoparticles as a function of zeta potential. Biomaterials. 2007;28(31):4600-4607.

14. Chithrani BD, Ghazani AA, Chan WC. Determining the size and shape dependence of gold nanoparticle uptake into mammalian cells. Nano Lett. 2006;6(4):662-668.

15. Malam Y, Loizidou M, Seifalian AM. Liposomes and nanoparticles: nanosized vehicles for drug delivery in cancer. Trends Pharmacol Sci. 2009;30(11):592-599.

16. Mout R, Moyano DF, Rana S, Rotello VM. Surface functionalization of nanoparticles for nanomedicine. Chem Soc Rev. 2012;41(7): 2539-2544.

17. Cho K, Wang X, Nie S, Chen Z, Shin DM. Therapeutic nanoparticles for drug delivery in cancer. Clin Cancer Res. 2008;14(5): $1310-1316$.

18. Farokhzad OC, Jon S, Khademhosseini A, Tran T-NT, LaVan DA, Langer R. Nanoparticle-aptamer bioconjugates: a new approach for targeting prostate cancer cells. Cancer Res. 2004;64(21):7668-7672.

19. Farokhzad OC, Cheng J, Teply BA, et al. Targeted nanoparticleaptamer bioconjugates for cancer chemotherapy in vivo. Proc Natl Acad Sci U S A. 2006;103(16):6315-6320. 
20. Hu S-L, Niu K-Y, Sun J, Yang J, Zhao N-Q, Du X-W. One-step synthesis of fluorescent carbon nanoparticles by laser irradiation. J Mater Chem. 2009;19(4):484-488.

21. Ratzinger G, Agrawal P, Körner W, et al. Surface modification of PLGA nanospheres with Gd-DTPA and Gd-DOTA for high-relaxivity MRI contrast agents. Biomaterials. 2010;31(33):8716-8723.

22. Bisht S, Feldmann G, Soni S, et al. Polymeric nanoparticle-encapsulated curcumin ("nanocurcumin"): a novel strategy for human cancer therapy. J Nanobiotechnology. 2007;5(3):1-18.

23. Kim J, Lee JE, Lee SH, et al. Designed fabrication of a multifunctional polymer nanomedical platform for simultaneous cancer - targeted imaging and magnetically guided drug delivery. Adv Mater Deerfield. 2008;20(3):478-483.

24. Yu MK, Jeong YY, Park J, et al. Drug - loaded superparamagnetic iron oxide nanoparticles for combined cancer imaging and therapy in vivo. Angew Chem Int Ed. 2008;47(29):5362-5365.

25. Albanese A, Lam AK, Sykes EA, Rocheleau JV, Chan WC. Tumouron-a-chip provides an optical window into nanoparticle tissue transport. Nat Comm. 2013;4:2718.

26. Goodman TT, Olive PL, Pun SH. Increased nanoparticle penetration in collagenase-treated multicellular spheroids. Int $J$ Nanomedicine. 2007;2(2):265.

27. Jeong JH, Byun Y, Park TG. Synthesis and characterization of poly(Llysine)-g-poly(D,L-lactic-co-glycolic acid) biodegradable micelles. J Biomater Sci Polymer Ed. 2003;14(1):1-11.

28. Gaumet M, Gurny R, Delie F. Fluorescent biodegradable PLGA particles with narrow size distributions: preparation by means of selective centrifugation. Int J Pharm. 2007;342(1-2):222-230.

29. Cui F, Shi K, Zhang L, Tao A, Kawashima Y. Biodegradable nanoparticles loaded with insulin-phospholipid complex for oral delivery: preparation, in vitro characterization and in vivo evaluation. J Control Release. 2006;114(2):242-250.

30. Panyam J, Labhasetwar V. Biodegradable nanoparticles for drug and gene delivery to cells and tissue. Adv Drug Deliv Rev. 2003;55(3): 329-347.

31. Anderson JM, Shive MS. Biodegradation and biocompatibility of PLA and PLGA microspheres. Adv Drug Deliv Rev. 2012;64(suppl 0): 72-82.

32. Bala I, Hariharan S, Kumar MR. PLGA nanoparticles in drug delivery: the state of the art. Crit Rev Ther Drug Carrier Syst. 2004;21(5):387-422.

33. Zalipsky S. Polyethylene glycol-lipid conjugates. Stealth Liposomes. Lasic DD, Martin FJ, editors. Boca Raton, FL: CRC Press; 1995:93-102.

34. Che J, Okeke C, Hu Z, Xu J. DSPE-PEG: a distinctive component in drug delivery system. Curr Pharm Des. 2015;21(12):1598-1605.

35. Wang R, Xiao R, Zeng Z, Xu L, Wang J. Application of poly(ethylene glycol)-distearoylphosphatidylethanolamine (PEG-DSPE) block copolymers and their derivatives as nanomaterials in drug delivery. Int $J$ Nanomedicine. 2012;7:4185-4198.

36. Makadia HK, Siegel SJ. Poly lactic-co-glycolic acid (PLGA) as biodegradable controlled drug delivery carrier. Polymers. 2011;3(3): 1377-1397.

37. Magut PKS, Das S, Fernand VE, et al. Tunable cytotoxicity of rhodamine 6G via anion variations. J Am Chem Soc. 2013;135(42): 15873-15879.
38. Fearon KCH, Plumb JA, Burns HJG, Calman KC. Reduction of the growth rate of the walker 256 tumor in rats by Rhodamine $6 \mathrm{G}$ together with hypoglycemia. Cancer Res. 1987;47(14):3684-3687.

39. Alexis F, Pridgen E, Molnar LK, Farokhzad OC. Factors affecting the clearance and biodistribution of polymeric nanoparticles. Mol Pharm. 2008;5(4):505-515.

40. Skwarczynski M, Hayashi Y, Kiso Y. Paclitaxel prodrugs: toward smarter delivery of anticancer agents. J Med Chem. 2006;49(25):7253-7269.

41. Kunz-Schughart LA, Kreutz M, Knuechel R. Multicellular spheroids: a three-dimensional in vitro culture system to study tumour biology. Int J Exp Pathol. 1998;79(1):1-23.

42. Keller GM. In vitro differentiation of embryonic stem cells. Curr Opin Cell Biol. 1995;7(6):862-869.

43. Kelm JM, Timmins NE, Brown CJ, Fussenegger M, Nielsen LK. Method for generation of homogeneous multicellular tumor spheroids applicable to a wide variety of cell types. Biotechnol Bioeng. 2003;83(2):173-180.

44. Fang RH, Aryal S, Hu C-MJ, Zhang L. Quick synthesis of lipid-polymer hybrid nanoparticles with low polydispersity using a single-step sonication method. Langmuir. 2010;26(22):16958-16962.

45. Ip S, MacLaughlin CM, Gunari N, Walker GC. Phospholipid membrane encapsulation of nanoparticles for surface-enhanced raman scattering. Langmuir. 2011;27(11):7024-7033.

46. Freitas C, Müller RH. Effect of light and temperature on zeta potential and physical stability in solid lipid nanoparticle (SLN $\left.{ }^{\mathrm{TM}}\right)$ dispersions. Int J Pharm. 1998;168(2):221-229.

47. Xu P, Gullotti E, Tong L, et al. Intracellular drug delivery by poly(lacticco-glycolic acid) nanoparticles, revisited. Mol Pharm. 2009;6(1): 190-201.

48. Zhang L, Chan JM, Gu FX, et al. Self-assembled lipid-polymer hybrid nanoparticles: a robust drug delivery platform. ACS Nano. 2008;2(8): 1696-1702.

49. Petersen S, Fahr A, Bunjes H. Flow cytometry as a new approach to investigate drug transfer between lipid particles. Mol Pharm. 2010;7(2): 350-363.

50. Aravind A, Jeyamohan P, Nair R, et al. AS1411 aptamer tagged PLGAlecithin-PEG nanoparticles for tumor cell targeting and drug delivery. Biotechnol Bioeng. 2012;109(11):2920-2931.

51. Zhang S, Li J, Lykotrafitis G, Bao G, Suresh S. Size-dependent endocytosis of nanoparticles. Adv Mater Deerfield. 2009;21(4):419-424.

52. Takei K, Haucke V, Slepnev V, et al. Generation of coated intermediates of clathrin-mediated endocytosis on protein-free liposomes. Cell. 1998;94(1):131-141.

53. Cabral H, Matsumoto Y, Mizuno K, et al. Accumulation of sub-100 nm polymeric micelles in poorly permeable tumours depends on size. Nat Nano. 2011;6(12):815-823.

54. Basaran C, Karcaaltincaba M, Akata D, et al. Fat-containing lesions of the liver: cross-sectional imaging findings with emphasis on MRI Am J Roentgenol. 2005;184(4):1103-1110.

55. Yoo D, Lee J-H, Shin T-H, Cheon J. Theranostic magnetic nanoparticles. Acc Chem Res. 2011;44(10):863-874.

56. Kabanov AV, Batrakova EV, Miller DW. Pluronic ${ }^{\circledR}$ block copolymers as modulators of drug efflux transporter activity in the blood-brain barrier. Adv Drug Deliv Rev. 2003;55(1):151-164. 


\section{Supplementary materials Nanoparticle test solution size \\ distributions}

Generally, a standard deviation of below 5\% is considered to be a monodispersed formulation ${ }^{1}$ and standard deviations of $5 \%-10 \%$ are considered to be narrowly dispersed. ${ }^{2}$ Although the nanoparticle solutions used in this study were not completely monodispersed, it is important to note that the goal of the study was not the characterization of the sizedependent uptake behavior of the MCTS of different cell lines. The aim was to show that using the size-dependent behavior of these MCTS, uptake, and penetration into tumors can be optimized. The size distributions of each nanoparticle solutions were specifically chosen as they had minimal overlap with each other in order to illustrate this concept (Figure S1).

\section{Positive controls: uptake and penetration studies}

As a positive control, MCTS models of PANC-1 and MIA PaCa-2 cell lines were incubated with free Rhodamine $6 \mathrm{G}$ fluorescent dye at a concentration of $0.5 \mu \mathrm{g} / \mathrm{mL}$, which was the same concentration as loaded in the multifunctional nanoparticles. Their relative intensities over a 24 -hour period as well as the bright field, fluorescent and intensity mapping images are presented in Figure S2. Uniform distribution of the nanoparticles was observed in the MCTS models of both cell lines after 24 hours of incubation (Figure S2C and F).

\section{Paclitaxel and Rhodamine 6G Release at $4^{\circ} \mathrm{C}$}

To investigate the long-term stability of the nanoparticles under storage condition, the nanoparticles were dialyzed
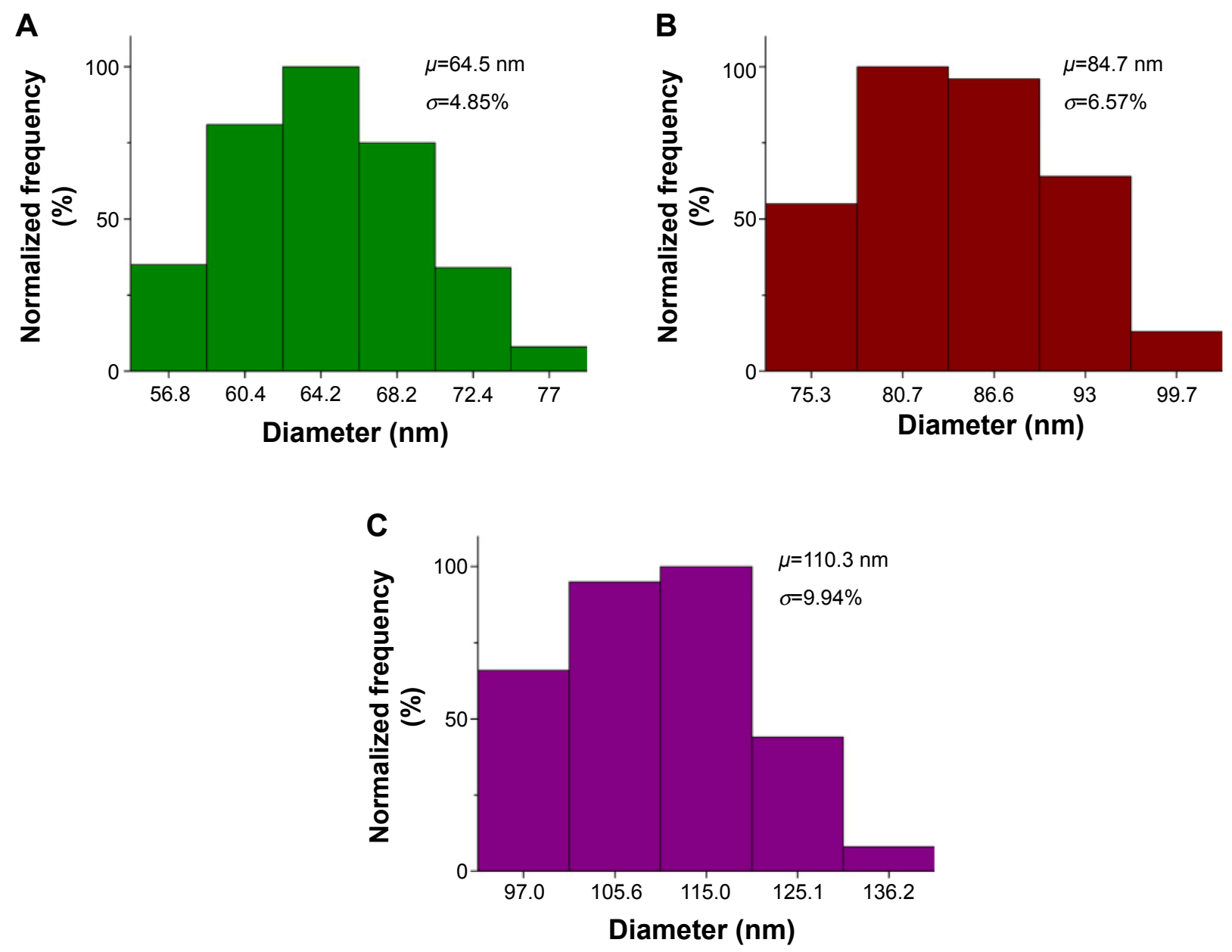

Figure SI Size distribution histograms of the nanoparticle test solutions used.

Notes: (A) Histogram for $65 \mathrm{~nm}$ sized nanoparticle solution. (B) Histogram for $85 \mathrm{~nm}$ sized nanoparticle solution. (C) Histogram for II 0 nm sized nanoparticle solution. $\mu$ represents the mean and $\sigma$ represents the standard deviation. 


\section{A}

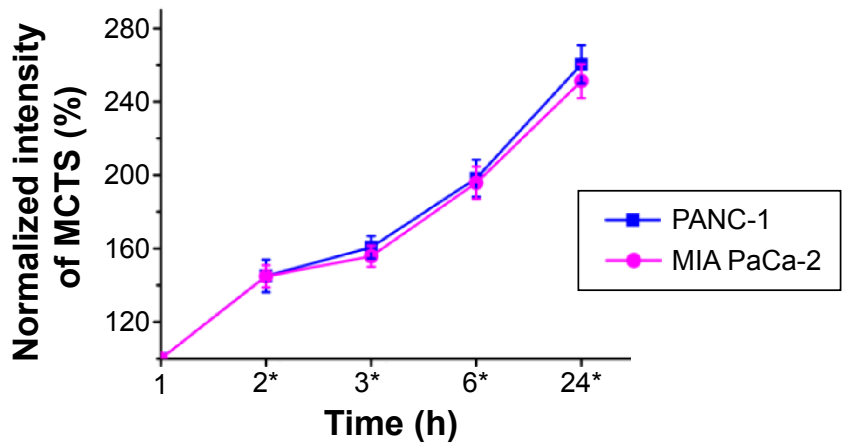

Relative fluorescent intensity (au)

$\overbrace{500} \underset{1,500}{1} \underbrace{1}_{3,500}$

$1 \mathrm{~h}$

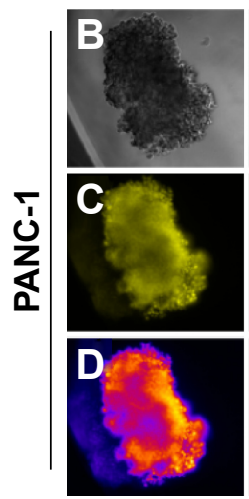

$6 \mathrm{~h}$

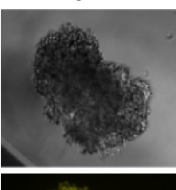

$24 \mathrm{~h}$
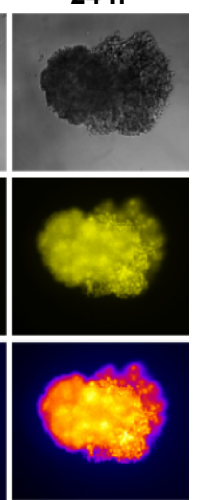

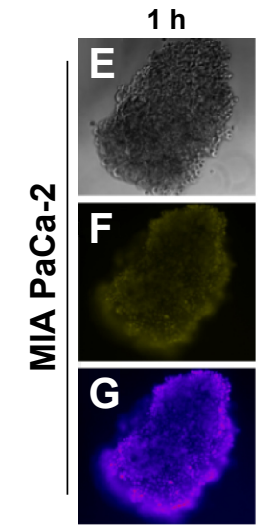

$6 \mathrm{~h}$

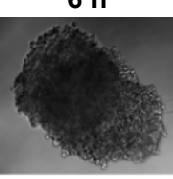

$24 \mathrm{~h}$
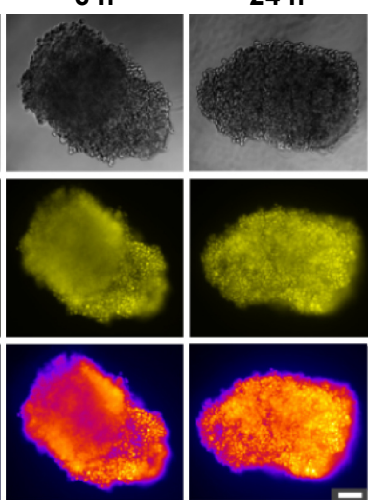

Figure S2 Positive control illustrating the uptake of free Rhodamine $6 \mathrm{G}(0.5 \mu \mathrm{g} / \mathrm{mL})$ in the MCTS of the PANC-I and MIA PaCa-2 cell lines

Notes: (A) Normalized fluorescence intensity of the MCTS of the PANC-I and MIA PaCa-2 cell lines at different time intervals. For MCTS models of PANC-I cell line incubated with free Rhodamine 6G at I hour, 6 hours, and 24 hours, (B) bright field images, (C) fluorescence images, and (D) intensity maps showing the relative fluorescent intensity of different regions of the MCTS of PANC-I. For the MCTS of the MIA PaCa-2 cell line incubated with free Rhodamine 6G at I hour, 6 hours, and 24 hours, (E) bright field images, (F) fluorescence images, and (G) intensity maps showing the relative fluorescent intensity of different regions of the MCTS of MIA PaCa-2. The scale bar represents $100 \mu \mathrm{m}$. Error bars indicate the standard deviation and the number of samples used in each measurement $(\mathrm{n}=8)$. *Indicates statistical difference $(P<0.05)$ between the fluorescent measurements at that time point of each cell line, based on the single variable analysis of variance test. No statistically significant difference was found between the fluorescent values of each cell line.

Abbreviations: MCTS, multicellular tumor spheroids; $h$, hours.

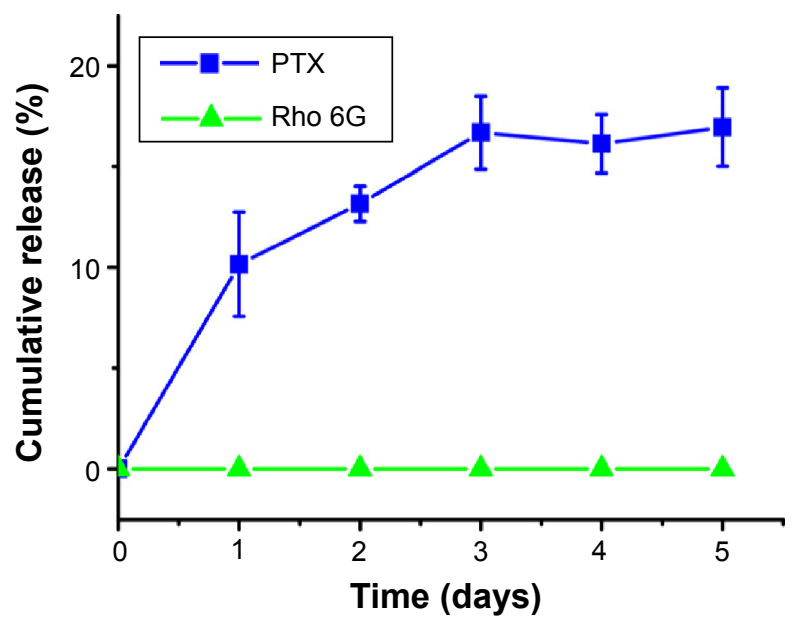

Figure S3 Paclitaxel (PTX) and Rhodamine 6G (Rho 6G) release at $4^{\circ} \mathrm{C}$ for all the three particle sizes (65 nm, $85 \mathrm{~nm}$, and I I0 nm) in PBS. Abbreviation: PBS, phosphate buffered saline. 
against PBS solution at $4^{\circ} \mathrm{C}$ in PBS solution. An initial, gradual release of $17.0 \%$ of Paclitaxel was observed in the first 3 days and, subsequently, no additional Paclitaxel was released on day 4 and 5. No Rhodamine $6 \mathrm{G}$ release was detected (Figure S3).

\section{References}

1. Sun S, Murray C, Weller D, Folks L, Moser A. Monodisperse FePt nanoparticles and ferromagnetic FePt nanocrystal superlattices. Science. 2000; 287(5460):1989-1992.

2. Park J, Joo J, Kwon SG, Jang Y, Hyeon T. Synthesis of monodisperse spherical nanocrystals. Angewandte Chemie Int Ed. 2007;46(25): $4630-4660$.

\section{Publish your work in this journal}

The International Journal of Nanomedicine is an international, peerreviewed journal focusing on the application of nanotechnology in diagnostics, therapeutics, and drug delivery systems throughout the biomedical field. This journal is indexed on PubMed Central, MedLine, CAS, SciSearch ${ }^{\circledR}$, Current Contents ${ }^{\circledR} /$ Clinical Medicine,
Journal Citation Reports/Science Edition, EMBase, Scopus and the Elsevier Bibliographic databases. The manuscript management system is completely online and includes a very quick and fair peer-review system, which is all easy to use. Visit http://www.dovepress.com/ testimonials.php to read real quotes from published authors. 